\title{
On the Closed-Form Solution to the Endogenous Growth Model with Habit Formation
}

\author{
Ryoji Hiraguchi \\ Faculty of Economics, Ritsumeikan University, Kyoto, Japan \\ Email: rhira@fc.ritsumei.ac.jp
}

Received June 25, 2012; revised July 23, 2012; accepted August 20, 2012

\begin{abstract}
We study the AK growth model with external habit formation. We show that there exists a unique solution path expressed in terms of the Gauss hypergeometric function. Using the closed-form solution, we also show that the optimal path converges to a balanced growth path.
\end{abstract}

Keywords: Endogenous Growth; Closed-Form Solution; Habit Formation

\section{Introduction}

The Gauss hypergeometric functions are typically used in mathematical physics, but are not so common in economics. As far as we know, Boucekkine and RuizTamarit [1] are the first to find that the functions are also useful in dynamic macroeconomics. They obtain an explicit solution path to Lucas-Uzawa two-sector endogenous growth model by using the hypergeometric function. They express the optimal path as a system of four differential equations and two transversality conditions and then use the hypergeometric functions for solving the system of equations.

Several authors get analytical solution paths to the exogenous growth models. Pérez-Barahona [2] investigates the model with non-renewable energy resources and find that the optimal path has a closed form solution path by using the hypergeometric function. Hiraguchi [3] finds that a solution path to the neoclassical growth model with endogenous labor is also represented by the special function.

Boucekkine and Ruiz-Tamarit [1] argue (see page 34) that the hypergeometric functions will also be useful in the investigation of the endogenous growth models. They guess that the transition dynamics of the models will be easier to understand if we can use the special functions. However, there is only a few literature that applies the special functions to the endogenous growth models other than the Lucas-Uzawa model. One example is Guerrini [4] who uses the special functions and obtains a closedform solution path to the AK model with logistic population growth. Broad applicability of the hypergeometric functions to the endogenous growth models is uncertain at this point, and more investiga- tions are needed.

In this paper, we study the AK endogenous model with external habit formation. The model has been investigated by many authors including Carroll et al. [5] and Gómez [6]. The utility function of the agent depends on both the absolute level of consumption and the ratio between consumption and habit stock. Here the habit formation is external and the level of the habit stock is exogenous to each agent. We show that there exists a unique solution path and it is represented by the hypergeometric function.

Habit formation in consumption is now popular in modern macroeconomics. Authors have explained some empirical facts by incorporating habits into the dynamic macroeconomic models. Abel [7] and Gal [8] show that habit formation can solve the equity premium puzzle in asset pricing models and Carroll et al. [9] provide an explanation of strong correlations between saving and growth. Some authors characterize the properties of the optimal paths in these models. Alvarez-Cuadrado et al. [10], Alonso-Carrera et al. [11] and Gómez [6] investigate the transitional dynamics and the stability of the optimal paths in endogenous growth models with habits, both analytically and numerically.

The problem of the previous papers is that they assume the existence and the uniqueness of the optimal path without proof. These properties are not at all obvious here, because there exists no general theorem on the existence of a solution path in an infinite horizons optimization problem with externalities. Here we utilize the special functions to show that their assumptions are in fact correct.

The note is organized as follows. Section 2 describes 
the model and obtains the first order conditions. Section 3 obtains the closed-from solution path. The conclusions are in Section 4. Proofs of the propositions are in Appendix.

\section{Set-Up}

In this section, we construct the one-sector endogenous growth model with external habit formation and obtains the first order conditions. There is a continuum of agents with unit measure. There is no population growth. The instantaneous utility function of each agent is

$$
u\left(c_{t}, h_{t}\right)=\frac{\left\{c_{t}^{1-\theta} \cdot\left(c_{t} / h_{t}\right)^{\theta}\right\}^{1-\sigma}-1}{1-\sigma}=\frac{\left(c_{t} h_{t}^{-\theta}\right)^{1-\sigma}-1}{1-\sigma} .
$$

Here $c_{t}$ is his own consumption, $h_{t}$ is the habit stock, $\sigma>0$ is the parameter on the utility curvature and $\theta \in(0,1)$ shows the importance of relative consumption level $\left(c_{t} / h_{t}\right)$ on the utility function. When there $\theta=0$ and the utility is time-separable, the parameter $\sigma$ coincides with the coefficient of the relative risk aversion. The habit stock is exogenous to the consumer and is accumulated by the following differential equation:

$$
\dot{h}_{t}=\beta\left(\bar{c}_{t}-h_{t}\right) .
$$

Here $\bar{c}_{t}$ is the average level of consumption and $\beta>0$ is a parameter. The parameter $\beta$ is high, the habit stock responds to the recent consumption quickly. The consumer solves the following problem:

$$
(P): \max _{c_{t}, k_{t}} \int_{0}^{\infty} e^{-\rho t} u\left(c_{t}, h_{t}\right) \mathrm{d} t, \text { s.t. } \dot{k}_{t}=A k_{t}-c_{t} \text {. }
$$

here $\rho>0$ is the discount factor, Equation (2) is the resource constraint, $k_{t}$ is physical capital and $A>0$ is the technology parameter. We assume that there is no capital depreciation. The initial capital stock $k_{0}$ and the initial habit stock $h_{0}$ are given. In what follows, we denote the growth rate of a variable $x_{t}$ as $\hat{x}_{t}\left(=\dot{x}_{t} / x_{t}\right)$.

The current value Hamiltonian is

$$
H=\left\{\left(c_{t} h_{t}^{-\theta}\right)^{1-\sigma}-1\right\} /(1-\sigma)+\lambda_{t}\left(A k_{t}-c_{t}\right) \text { where } \lambda_{t}
$$

is the multiplier. The first order conditions (FOCs) and the transversality condition (TC) are

$$
\begin{gathered}
\operatorname{FOC}(\mathrm{k}): \frac{\dot{\lambda}_{t}}{\lambda_{t}}=\rho-A, \\
\operatorname{FOC}(\mathrm{c}): c_{t}^{-\sigma} h_{t}^{-\theta(1-\sigma)}=\lambda_{t}, \\
\mathrm{TC}: \lim _{t \rightarrow \infty}\left(\lambda_{t} k_{t} e^{-\rho t}\right)=0 .
\end{gathered}
$$

Here FOC $(x)$ means the FOC on the variable $x$. In equilibrium, the individual consumption $c_{t}$ is equal to the average consumption $\overline{c_{t}}$ and the habit stock is accu- mulated according to

$$
\dot{h}_{t}=\beta\left(c_{t}-h_{t}\right) .
$$

The path $\left\{c_{t}, k_{t}, h_{t}\right\}$ is optimal if and only if it satisfies Equations (2)-(6) for some $\lambda_{t} \geq 0$.

The next lemma shows that when the productivity $A$ is too high, the interior optimal path does not exist ${ }^{1}$.

Lemma 1. If $A(1-\theta)(1-\sigma)>\rho$, the optimal path does not exists.

Proof. See the Appendix. $\square$

Moreover, if the productivity is too low and satisfies $\rho>A$, Equations (3) and (4) together imply that the balanced growth rate of consumption (and also habit stock) is negative. Thus we impose the following restriction on the parameters to ensure that the optimal path is interior and that the optimal growth rate is positive:

$$
A(1-\theta)(1-\sigma)<\rho<A \text {. }
$$

\section{Closed-Form Solution}

To characterize the optimal path, we first obtain a linear differential equation on the habit-consumption ratio $z_{t}=h_{t} / c_{t}$. Note that $\hat{z}_{t}=\hat{h}_{t}-\hat{c}_{t}$. Substitution of Equation (4) into Equation (3) yields

$$
\sigma \hat{z}_{t}-\gamma \hat{h}_{t}=\rho-A \text {. }
$$

where $\gamma=(1-\theta) \sigma+\theta>0$. On the other hand, Equation (6) is written as $\hat{h}_{t}=\beta\left(1 / z_{t}-1\right)$. Thus Equation (8) implies $\sigma \hat{z}_{t}=\gamma \beta\left(1 / z_{t}-1\right)-(A-\rho)$. Multiplying both sides of the equation by $Z_{t}$, we get a linear differential equation $\dot{z}_{t}=-\omega\left(z_{t}-z^{*}\right)$ with

$\omega=(A-\rho+\gamma \beta) / \sigma>0$ and $z^{*}=\beta \gamma /(A-\rho+\gamma \beta)>0$. The solution is

$$
z_{t}=z^{*}+e^{-\omega t}\left(z_{0}-z^{*}\right) .
$$

Since $\omega>0$, the habit consumption ratio $z_{t}$ converges to $z^{*}$ as $t$ goes to $\infty$.

Next we use Equation (9) to obtain the equilibrium consumption path. Since $\hat{c}_{t}+\hat{z}_{t}=\hat{h}_{t}$, Equation (8) is reexpressed as $(\sigma-\gamma) \hat{z}_{t}=\gamma \hat{c}_{t}+\rho-A$. Thus

$$
\left(c_{t} / c_{0}\right)^{\gamma}=e^{(A-\rho) t}\left(z_{t} / z_{0}\right)^{\sigma-\gamma} \text { and the consumption } C_{t} \text { is }
$$

$$
c_{t}=e^{g t} z_{t}^{\sigma / \gamma-1}\left(z_{0}^{-\sigma / \gamma} h_{0}\right),
$$

where the parameter $g$ is defined as

$$
g=\frac{A-\rho}{\gamma}=\frac{A-\rho}{(1-\theta) \sigma+\theta}>0 .
$$

Note that by definition, $c_{0}=h_{0} / z_{0}$. Since $\lim _{t \rightarrow \infty} Z_{t}=z^{*}<\infty$, the consumption growth rate converges to $g$. Later we show that the growth rate of the

${ }^{1}$ It is not a simple task to prove Lemma 1 only by using the transversality condition (5). This is because we cannot easily obtain the asymptotic growth rate of physical capital by using the system of the differential Equations (2)-(6). 
equilibrium capital also converges to the same value $g$.

In Equation (10), $z_{0}$ is unknown. To fix its value, we have to use the transversality condition (5). Here the resource constraint (2) can be written as $k_{t}=e^{A t}\left(k_{0}-\int_{0}^{t} e^{-A s} c_{s} \mathrm{~d} s\right)$. Using Equation (10), we get

$$
k_{t}=e^{A t}\left[k_{0}-z_{0}^{-\sigma / \gamma} h_{0} \int_{0}^{t} e^{-(A-g) s} z_{s}^{\sigma / \gamma-1} \mathrm{~d} s\right] .
$$

Under the parametric restriction (7), $A(1-\gamma)=A(1-\theta)(1-\sigma)<\rho$ and then $A>g=(A-\rho) / \gamma$. Thus the term $\int_{0}^{t} e^{-(A-g) s} z_{s}^{\sigma / \gamma-1} \mathrm{~d} s$ converges to a finite constant as $t$ goes to $\infty$. The next lemma simplifies the transversality condition (5).

Lemma 2. The transversality condition (5) holds if and only if

$$
k_{0}=z_{0}^{-\sigma / \gamma} h_{0} \int_{0}^{\infty} e^{-(A-g) s} z_{s}^{\sigma / \gamma-1} \mathrm{~d} s .
$$

There exists a unique $z_{0}$ that satisfies the condition. (Here $k_{0}$ and $h_{0}$ are given.)

Proof. See the Appendix. $\square$

Using Lemma 2, we can re-write the equilibrium capital as

$$
k_{t}=e^{A t} z_{0}^{-\sigma / \gamma} h_{0} \int_{t}^{\infty} e^{-(A-g) s}\left(z_{s}\right)^{\sigma / \gamma-1} \mathrm{~d} s .
$$

We now express the equilibrium capital without using the integral. As Hiraguchi (2012) shows, the hypergeometric function

$$
{ }_{2} \mathrm{~F}_{1}(a, b, c ; z)=\sum_{n=0}^{\infty}\left\{(a)_{n}(b)_{n} /(c)_{n}\right\} z^{n} / n !
$$

with $(a)_{n}=\Gamma(a+n) / \Gamma(a)$ satisfies

$$
\begin{aligned}
& \int_{x}^{\infty} e^{-a_{1} y}\left(b_{1}+b_{2} e^{-a_{2} y}\right)^{a_{3}} \mathrm{~d} y \\
= & \frac{e^{-a_{1} x} b_{1}^{a_{3}}}{a_{1}}{ }_{2} \mathrm{~F}_{1}\left(-a_{3}, \frac{a_{1}}{a_{2}}, 1+\frac{a_{1}}{a_{2}} ;-\frac{b_{2}}{b_{1}} e^{-a_{2} x}\right),
\end{aligned}
$$

where $a_{1}>0, a_{2}>0, a_{3}>0, b_{1}>0$, and $b_{2}$ are constant ${ }^{2}$. A similar equation is also proved by Boucekkine and Ruiz-Tamarit [1] (see Proposition 1 in page 40). Thus we can express the integral part of $k_{t}$ as

$$
\begin{aligned}
& \int_{t}^{\infty} e^{-(A-g) s} z_{s}^{\sigma / \gamma-1} \mathrm{~d} s \\
= & \frac{\left(z^{*}\right)^{\sigma / \gamma-1}}{A-g} e^{-(A-g) t}{ }_{2} \mathrm{~F}_{1} \\
& \times\left(1-\frac{\sigma}{\gamma}, \frac{A-g}{\omega}, 1+\frac{A-g}{\omega} ; \frac{z^{*}-z_{0}}{z^{*}} e^{-\omega t}\right) .
\end{aligned}
$$

${ }^{2}$ If we let $z=e^{-a_{1} y}$, we can easily show that the integral is equal to $a_{1}^{-1} b_{2}^{a_{3}} \int_{0}^{e^{-a_{1} x}}\left(b_{1} / b_{2}+z^{\left(a_{2} / a_{1}\right)}\right)^{a_{3}} \mathrm{~d} z$. As many authors have already shown, the integral $\int\left(a+z^{b}\right)^{c} \mathrm{~d} z$ can be obtained by using the hypergeometric function.
Recall that $z_{t}=z^{*}+e^{-\omega t}\left(z_{0}-z^{*}\right)$. Finally we get the following proposition.

Proposition 1. The optimal path $\left\{c_{t}, h_{t}, k_{t}\right\}$ exists, is unique and is expressed as

$$
\begin{gathered}
c_{t}=e^{g t}\left\{z^{*}+e^{-\omega t}\left(z_{0}-z^{*}\right)\right\}^{\sigma / \gamma-1}\left(z_{0}\right)^{-\sigma / \gamma} h_{0}, \\
h_{t}=z_{t} c_{t}=e^{g t}\left\{z^{*}+e^{-\omega t}\left(z_{0}-z^{*}\right)\right\}^{\sigma / \gamma}\left(z_{0}\right)^{-\sigma / \gamma} h_{0}, \\
k_{t}=e^{g t}{ }_{2} \mathrm{~F}_{1}\left(1-\frac{\sigma}{\gamma}, \frac{A-g}{\omega}, 1+\frac{A-g}{\omega} ; \frac{z^{*}-z_{0}}{z^{*}} e^{-\omega t}\right) \frac{\left(z^{*}\right)^{\sigma / \gamma-1} h_{0}}{\left(z_{0}\right)^{\sigma / \gamma}(A-g)} .
\end{gathered}
$$

The parameters are $\gamma=(1-\theta) \sigma+\theta>0$, $\omega=(A-\rho+\gamma \beta) / \sigma>0, \quad z^{*}=\beta \gamma /(A-\rho+\gamma \beta)>0$ and $g=(A-\rho) /\{(1-\theta) \sigma+\theta\}$. The value of $Z_{0}$ is determined by Equation (12).

It is known that for any $a, b$ and $c,{ }_{2} \mathrm{~F}_{1}(a, b, c ; 0)=1$. Thus the growth rate of the capital also converges to $g$. Therefore the optimal path $\left\{c_{t}, h_{t}, k_{t}\right\}$ always converges to a balanced growth path with the growth rate $g$.

If $\theta=0$ (no habit formation), then $\sigma=\gamma$ and the first term of the hypergeometric function in Equation (16) is $1-\sigma / \gamma=0$. Thus the optimal consumption path and capital path grows exponentially:

$$
\begin{gathered}
c_{t}=\left(z_{0}\right)^{-\sigma / \gamma} h_{0} e^{g t}, \\
k_{t}=\frac{\left(z^{*}\right)^{\sigma / \gamma-1} h_{0}}{\left(z_{0}\right)^{\sigma / \gamma}(A-g)} e^{g t} .
\end{gathered}
$$

It is well-known that the basic AK growth model does not have transitional dynamics and the optimal growth rate is always constant.

\section{Conclusion}

In this paper, we obtain a closed-form solution path to the AK growth model with habit formation. As Boucekkine and Ruiz-Tamarit [1] claim, the hypergeometric functions are actually very useful in the investigation of the endogenous growth models. We guess that the Gauss hypergeometric functions are applicable to many kinds of the dynamic macroeconomic models. As a future study, we hope to investigate the different kinds of the endogenous growth models, especially the growth models with R \& D.

\section{REFERENCES}

[1] R. Boucekkine and R. Ruiz-Tamarit, "Special Functions for the Study of Economic Dynamics: The Case of the Lucas-Uzawa Model," Journal of Mathematical Economics, Vol. 44, No. 1, 2008, pp. 33-54. doi:10.1016/j.jmateco.2007.05.001

[2] A. Pérez-Barahona, "Nonrenewable Energy Resources as 
Input for Physical Capital Accumulation: A New Approach,” Macroeconomic Dynamics, Vol. 15, No. 1, 2011, pp. 1-30. $\underline{\text { doi:10.1017/S1365100509090415 }}$

[3] R. Hiraguchi, "A Note on the Analytical Solution to the Neoclassical Growth Model with Leisure," Macroeconomic Dynamics, 2012, pp. 1-7. doi:10.1017/S1365100512000442

[4] L. Guerrini, "Transitional Dynamics in the Ramsey Model with AK Technology and Logistic Population Change," Economics Letters, Vol. 109, No. 1, 2010, pp. 17-19. doi:10.1016/j.econlet.2010.07.002

[5] C. Carroll, J. Overland and D. Weil, "Comparison Utility in a Growth Model,” Journal of Economic Growth, Vol. 2, No. 4, 1997, pp. 339-367. doi:10.1023/A:1009740920294

[6] M. A. Gómez, "Convergence Speed in the AK Endogenous Growth Model with Habit Formation,” Economics Letters, Vol. 100, No. 1, 2008, pp. 16-21. doi:10.1016/j.econlet.2007.10.022

[7] A. Abel, “Asset Prices under Habit Formation and Catch- ing Up with the Joneses,” American Economic Review, Vol. 80, No. 2, 1990, pp. 38-42.

[8] J. Gal, "Keeping up with the Joneses: Consumption Externalities, Portfolio Choice, and Asset Prices," Journal of Money, Credit, and Banking, Vol. 26, No. 1, 1994, pp. 18. doi:10.2307/2078030

[9] C. Carroll, J. Overland and D. Weil, "Saving and Growth with Habit Formation,” American Economic Review, Vol. 90, No. 3, 2000, pp. 341-355. doi:10.1257/aer.90.3.341

[10] F. Alvarez-Cuadrado, G. Monteiro and S. Turnovsky, "Habit Formation, Catching up with the Joneses, and Economic Growth,” Journal of Economic Growth, Vol. 9, No. 1, 2004, pp. 47-80. doi:10.1023/B:JOEG.0000023016.26449.eb

[11] J. Alonso-Carrera, J. Caballé and X. Raurich, "Growth, Habit Formation, and Catching up with the Joneses," European Economic Review, Vol. 49, No. 6, 2005, pp. 16651691.doi:10.1016/j.euroecorev.2004.03.005

\section{Appendix}

\section{Proof of Lemma 1}

Let $\varepsilon=A(1-\theta)(1-\sigma)-\rho>0$. Consider a path such that $c_{t}=(\varepsilon / 2) k_{t}>0$. The path is feasible since the resource constraint can be written as $\dot{k}_{t} / k_{t}=(A-\varepsilon / 2)>0$. Along the path, we can easily show that

$$
\lim _{t \rightarrow \infty} \hat{h}_{t}=\lim _{t \rightarrow \infty} \hat{c}_{t}=\lim _{t \rightarrow \infty} \hat{k}_{t}=A-\varepsilon / 2 .
$$

If we denote the instantaneous utility as

$$
U_{t}=e^{-\rho t}\left\{\left(c_{t} h_{t}^{-\theta}\right)^{1-\sigma}-1\right\} /(1-\sigma),
$$

then the intertemporal utility is equal to $\int_{0}^{\infty} U_{t} \mathrm{~d} t$. The growth rate of $U_{t}$ converges to a positive constant because

$$
\lim _{t \rightarrow \infty} \hat{U}_{t}=-\rho+(1-\sigma)(1-\theta)(A-\varepsilon / 2)=\varepsilon / 2>0 .
$$

Thus $\int_{0}^{\infty} U_{t} \mathrm{~d} t=+\infty$.

Then the optimal path does not exist. $\square$

\section{Proof of Lemma 2}

We first show that Equation (12) is equivalent to Equation (5). The growth rate of the multiplier $\lambda_{t}$ is $\rho-A$ and then the term $\lambda_{t} k_{t} e^{-\rho t}$ can be expressed as $\lambda_{t} k_{t} e^{A t}$. Thus the transversality condition holds if and only if

$$
\lim _{t \rightarrow \infty}\left(e^{A t} k_{t}\right)=\lim _{t \rightarrow \infty}\left[k_{0}-z_{0}^{-\sigma / \gamma} h_{0} \int_{0}^{t} e^{-(A-g) s} z_{s}^{\sigma / \gamma-1} \mathrm{~d} s\right]=0 .
$$

Under Equation (7) $A>g$ and then $\int_{0}^{\infty} e^{-(A-g) s} z_{s}^{\sigma / \gamma-1} \mathrm{~d} s$ is finite. Thus the above equation is equivalent to Equation (12). We next show that there exists a unique $z_{0}$ satisfying Equation (12). If we define a function $G_{t}$ as

$$
G_{t}(z)=z^{-\sigma / \gamma}\left(e^{-\omega t} z+z^{*}\left(1-e^{-\omega t}\right)\right)^{\sigma / \gamma-1},
$$

Equation (12) is written as

$$
k_{0} / h_{0}=\int_{0}^{\infty} e^{-(A-g) t} G_{t}\left(z_{0}\right) \mathrm{d} t .
$$

Since $1-e^{-\omega t} \geq 0$, the function

$$
\begin{aligned}
& \qquad G_{t}(z)=\left\{e^{-\omega t} z+z^{*}\left(1-e^{-\omega t}\right)\right\}^{-1} \\
& \left\{e^{-\omega t}+z^{*}\left(1-e^{-\omega t}\right) / z\right\}^{\sigma / \gamma} \text { is a strictly decreasing } \\
& \text { function of } z \text {. Moreover, } G_{t}(0)=\infty \text { and } G_{t}(\infty)=0 \text {. } \\
& \text { Thus there exists a unique } z_{0} \text { satisfying } \\
& k_{0} / h_{0}=\int_{0}^{\infty} G_{t}\left(z_{0}\right) d t \text {. }
\end{aligned}
$$

